\title{
L'ennemi intérieur, de la guerre coloniale au contrôle sécuritaire
}

\section{Mathieu Rigouste}

\section{(2) OpenEdition \\ Journals}

Édition électronique

URL : http://journals.openedition.org/conflits/3128

DOI : $10.4000 /$ conflits.3128

ISSN : $1777-5345$

Éditeur :

CCLS - Centre d'études sur les conflits lilberté et sécurité, L'Harmattan

Édition imprimée

Date de publication : 15 novembre 2007

Pagination : 157-174

ISBN : 978-2-296-04582-8

ISSN : 1157-996X

Référence électronique

Mathieu Rigouste, "L'ennemi intérieur, de la guerre coloniale au contrôle sécuritaire », Cultures \&

Conflits [En ligne], 67 | automne 2007, mis en ligne le 04 janvier 2010, consulté le 30 mars 2021. URL http://journals.openedition.org/conflits/3128 ; DOI : https://doi.org/10.4000/conflits.3128 


\section{L'ennemi intérieur, de la guerre coloniale au contrôle sécuritaire}

\section{Mathieu RIGOUSTE}

Mathien Rigouste est chercheur en socio-histoire, il s'intéresse aux processus de légitimation du contrôle et aux mécanismes de représentation de la menace. Il a notamment travaillé sur la construction médiatique de l'immigration et publie sa thèse (début 2008) sur l'ennemi intérieur dans la pensée militaire : L'Ennemi intérieur postcolonial. De la guerre coloniale au contrôle sécuritaire (1954-2007), Paris, La Déconverte, 2008.

$\mathrm{F}_{\text {française }}{ }^{1}$ permet de montrer comment la pensée sécuritaire a été alimentée à partir de la reformulation d'un certain nombre de dispositifs de contrôle conçus pour, par et au travers de la guerre coloniale. En analysant la manière dont les courants dominants de la pensée militaire française ont conçu le contrôle de l'immigration, nous avons observé la reconstruction d'un ennemi intérieur socio-ethnique, la régénération puis la généralisation dans le temps et dans l'espace, à travers le modèle sécuritaire, d'une technologie conçue pour le contrôle exceptionnel de populations infériorisées : la lutte contre-subversive.

Nous allons nommer "pensée militaire » le réseau d'énoncés dominant le champ de production et de diffusion des discours dans l'institution militaire. Nous nous intéresserons en cela à des courants de pensée et non pas à la doctrine. Il s'agit de montrer comment certains énoncés parviennent à orienter l'institution pratique de la violence d'Etat à partir d'un multi-positionnement aux marges du discours officiel ou doctrinal et dans de multiples soussecteurs des champs de la production et de la légitimation du contrôle. Les étapes de la construction discursive de la menace migratoire montrent que celle-ci résulte de luttes et solidarités entre réseaux d'idées et qu'elle découle notamment de la montée en puissance et de la multipolarisation de réseaux

1. Etude réalisée au cours du doctorat. Voir Rigouste M., L'Ennemi intérieur postcolonial. De la lutte contre-subversive au contrôle de l'immigration dans la pensée militaire française. Une socio-bistoire du contrôle sécuritaire, 1954-2007, Kadri A. (dir.), université Paris-VIII, 2007. 
motivés par la remobilisation, aux côtés du concept de dissuasion, du répertoire de la subversion.

Les formations discursives mises en jeu par les grands médias de la communauté politico-militaire ${ }^{2}$ et par la principale structure civilo-militaire de "promotion de l'esprit de défense ${ }^{3}$ " permettent de reconstruire la généalogie de ces courants de pensée et d'étudier les mécanismes de lutte pour la reconnaissance de l'immigration comme menace. Nous avons pour cela isolé les idées et rhétoriques principales autour desquelles s'organisaient les énoncés observés à la fois dans le corpus de presse et dans les archives de l'IHEDN. Nous les avons classées en courants de pensée en situant les positions de leurs énonciateurs dans le champ et leurs interrelations. Pour chaque respiration historique, nous avons pu définir de deux à six positions principales, souvent inégales et entretenant des liens de solidarité et/ou de conflit.

L'histoire des pensées contre-subversives et celle de la construction de l'immigration comme menace mettent en évidence le fait que la désignation de l'immigration, de la délinquance ou des violences urbaines comme des menaces intéressant la défense nationale ont fourni un répertoire alternatif face à la disparition des menaces qui avaient permis de justifier la doctrine de défense globale ${ }^{4}$. Nous tentons de montrer que les technologies contre-subversives issues du terrain colonial, la lutte contre l'immigration et l'élaboration du contrôle sécuritaire ont finalement ceci en commun d'appeler à la coopération inter ou trans-nationale. La construction de l'immigration comme menace s'inscrit ainsi dans le cadre de la représentation d'une menace transversale et globale, susceptible de promouvoir la mise en œuvre d'un contrôle sécuritaire global.

2 . Nous avons, dans le cadre de notre thèse, lu, répertorié, classifié et analysé la très grande majorité des articles parus dans la revue Défense nationale depuis 1954, principale revue d'information du monde politico-militaire francais dont la rédaction est civilo-militaire, ainsi que l'ensemble des articles parus dans Défense, la revue de l'IHEDN (Institut des hautes études de défense nationale) depuis sa fondation en 1947. Nous avons eu recours à la Revue militaire d'information de manière moins systématique. Cela représente $10 \mathrm{~m}^{3}$ de documents parmi lesquels nous avons particulièrement analysé 300 articles.

3. Nous avons eu accès à l'ensemble du fonds IHEDN depuis 1947 (dossiers d'information aux auditeurs, rapports de comités, conférences, etc.). L'IHEDN est chargé de "promouvoir l'esprit de défense dans la nation ». Il organise pour cela différentes activités visant à former des " cadres de la nation » à l'esprit de défense et aux nécessités de sa diffusion dans la société. Son fonds d'archives donne accès aux documents distribués par la direction à des auditeurs, supposés les informer de l'état des questions politico-militaires importantes, l'ensemble des travaux rédigés par les comités d'auditeurs sur ces sujets et les synthèses élaborées par la direction de l'IHEDN afin d'informer le gouvernement sur l'état de la pensée des cadres de la nation sur les questions de défense.

4. Initiée principalement par les généraux André Beaufre et Jean De Lattre de Tassigny, convaincus de la nécessité de transformer radicalement l'appareil doctrinal français, la doctrine de la défense globale développe l'idée selon laquelle, confronté à une menace embrassant désormais l'ensemble de la société, le rôle du souverain est de mettre l'ensemble de la nation en état de veille militaire permanente, et d'assurer sa cohésion derrière le chef militaire, ceci en permettant à l'armée de pénétrer les sphères les plus intimes de l'organisation sociale. 


\section{La question postcoloniale. Repenser la subversion intérieure dans le contexte de la dissuasion}

La lutte contre-subversive émerge à l'intersection de la Guerre froide et des guerres coloniales. La question postcoloniale interroge ce qui a pu être conservé, évacué ou reformulé de ces dispositifs de contrôle à travers les configurations socio-historiques issues des guerres coloniales.

\section{La dissuasion contre la subversion}

L'installation de Charles De Gaulle au pouvoir en 1958 scelle une opposition entre deux réseaux dominants à l'intérieur de l'institution politicomilitaire. Ceux-ci s'organisent autour de deux idées contradictoires. Le premier considère qu'il est possible de reconduire les intérêts énergétiques, économiques et stratégiques français en Algérie à travers la mise en place d'un Etat algérien subordonné et d'une nouvelle forme de tutelle, la "Communauté française ». Le second s’organise autour des théoriciens et des techniciens de la doctrine de la guerre révolutionnaire pour imposer la souveraineté française et maintenir l'axe Paris-Brazzaville, en instituant un commandement militaire sur l'Algérie, censé être garant du non-encerclement par le monde communiste de la tête de pont OTAN que constituait l'Europe occidentale ${ }^{5}$. La genèse politico-militaire de la Ve République est en partie le fruit des luttes des réseaux gaullistes ou souverainistes pour dominer, contenir ou orienter celui des subversifs.

Considérant le militaire comme le chirurgien d'un corps national pourrissant, la doctrine de la guerre révolutionnaire et les théories contre-subversives justifient l'avancée du militaire sur le civil et le politique, et appellent à la mise en place de juridictions d'exception tendant à permettre au militaire de se déployer dans le cadre civil et policier ${ }^{6}$. Elle fournit en cela un projet de conquête de la société pour les réseaux qui la promeuvent et les industriels qui les soutiennent. Elle s'appuie notamment sur une utopie de société immunisée contre toute forme de désordre, une société militarisée. Les tentatives de coups d'Etat militaire de la fin de la guerre d'Algérie font prendre conscience au président De Gaulle de cette dimension. La doctrine, après avoir structuré toute la pensée et la pratique de la pacification dans la guerre d'Algérie, se voit ainsi évacuée du contrôle intérieur et employée dans la formation des Etats

5. Voir «La guerre révolutionnaire va-t-elle nous embraser ? ", rapport du Contrôleur général de l'armée Libermann, $1^{\text {er }}$ octobre 1955, ministère de la Défense nationale, nº03226 DN/CAB/EMP, le 19 janvier 1956.

6. Sur la doctrine de la guerre révolutionnaire, lire les thèses de Gabriel Périès et de Paul et MarieCatherine Villatoux : Périès G., De l'action militaire à l'action politique. Impulsion, codification et application de la doctrine de la "guerre révolutionnaire " au sein de l'armée française (1944-1960), thèse de science politique, Lagroye J. (dir.), Paris-I, 1999 ; Villatoux M.-C. et P., La Guerre et l'action psychologique en France, 1945-1960, thèse d'histoire, Paris-I, Vaïsse M. (dir.), 2002. 
postcoloniaux affiliés à la France et en direction des armées alliées 7 . Son externalisation répond en cela à la stratégie de la dissuasion, qui, en plaçant la France de manière indépendante dans l'équilibre nucléaire, devait lui permettre de relativiser la perte de l'empire.

Les pensées contre-subversives se sont alimentées d'une série d'allers et retours entre théâtres d'opérations et amphithéâtres, entre terrain et bureaux. Elles furent d'abord véhiculées par des officiers en opération en Indochine, puis en Algérie, et des cadres politico-militaires chargés de la réflexion et de l'enseignement sur la guérilla et la Guerre froide en métropole. Elles sont conçues par de jeunes officiers comme Charles Lacheroy et Roger Trinquier, dont le parcours retrace celui de la doctrine de la guerre révolutionnaire, depuis les maquis indochinois jusqu'aux amphithéâtres politico-militaires des pays de l'OTAN, physiquement ou à travers leurs ouvrages. Comme d'autres, restés anonymes, ces officiers furent tenus en échec puis vaincus par la guérilla Viet-Minh. Ils promeuvent à leur retour en métropole une analyse largement racialiste et anticommuniste du phénomène, teintée de rhétoriques émanant des travaux sur la foule issus de la psychologie sociale de l'entre-deux-guerres ${ }^{8}$. Ils proposent de renverser au profit des intérêts occidentaux les techniques d'emploi de la «terreur » observées en Indochine. Après avoir séduit la majorité des réseaux dominant la réflexion politico-militaire sur le « problème colonial » et orienté les activités des principales institutions chargées de diffuser la pensée militaire (CHEM, ESG, IHEDN, CEAA, etc.), ceux-ci sont propulsés par de plus vieux énonciateurs, dominant par ailleurs les courants principaux de la pensée politico-militaire et largement anticommunistes, tels que les généraux Ely, Beaufre, Allard, Boucherie, Le Hingrat, Nemo ou Hogard. Ces énoncés vont ainsi être dotés d'un statut doctrinal, officiel, légitime et orienter l'ensemble des pratiques de la violence militaro-policière jusqu'à la fin de la guerre d'Algérie ${ }^{9}$ et ce, à travers la mise en place de nombreux bureaux sur le terrain (5e bureau d'action psychologique, CIPCG d'Árzew, SAS, DOP, etc. $\left.{ }^{10}\right)$. La bataille d'Alger et le dispositif de protection urbain mis

7. Sur la formation des doctrines anglo-saxonnes de la counter-insurgency à partir des théories françaises, notamment les rapports entre Frank Kitson et Roger Trinquier, lire par exemple Faligot R., Guerre spéciale en Europe, Paris, Flammarion, 1980. Sur l'exportation du répertoire contre-subversif pour la formation des post-colonies africaines, on peut lire Périès G., Servenay D., Une guerre noire. Enquête sur les origines du génocide Rwandais (1959-1994), Paris, La Découverte, 2007.

8. A ce sujet lire Potier E., "Imaginaire du contrôle des foules dans l'armée de terre française ", Cultures E Conflits, n56, hiver 2004.

9. Voir par exemple les documents : commandant Hogard J., «L'armée française devant la guerre révolutionnaire ", Défense nationale, $1^{\mathrm{er}}$ semestre 1957, p. 77 ; général Allard J., commandant le Corps d'armée d'Alger, «Vérités sur l'Affaire algérienne », Défense nationale, janvier 1958, p. 5.

10. CIPCG : Centre d'instruction et de préparation à la contre-guérilla. DOP : Dispositif opérationnel de protection. SAS : Section administrative spécialisée. Sur l'ensemble de ces institutions, voir les travaux de Raphaëlle Branche : Branche R., La Torture et l'armée pendant la guerre d'Algérie, 1954-1962, Paris, Gallimard, coll. « La suite des temps », 2001. 
en place par le général Massu et le colonel Trinquier constituent, à ce titre, les expérimentations les plus approfondies et les plus médiatisées de la pratique de la guerre révolutionnaire.

On peut synthétiser les pensées contre-subversives à travers cinq propositions principales, dont la somme constitue une sorte de diagramme de la doctrine de la guerre révolutionnaire :

1. Les populations colonisées sont des milieux de prolifération de la menace communiste qu'il faut immuniser.

2. Le renseignement doit permettre de faire apparaître les hiérarchies parallèles adverses qu'il faut tenir, détruire ou remplacer.

3. La terreur permet de tenir la population, l'ennemi s'en sert, il faut tourner cette arme contre lui.

4. L'action psychologique permet de retourner la population tenue et la guerre psychologique de tromper l'ennemi.

5. Le quadrillage militaro-policier de l'espace urbain constitue un acte chirurgical radical pour immuniser la population subvertie.

L'histoire de la construction de l'ennemi intérieur postcolonial dans la pensée politico-militaire française s'enchevêtre avec celle de la restauration de la subversion aux côtés de la dissuasion, à son retour dans le cadre du contrôle intérieur. Les années 1960 constituent ainsi une phase d'imposition de la doctrine gaulliste de la dissuasion et de réduction des théories de la lutte contresubversive pour lesquelles la traque de l'ennemi intérieur et la gestion de la «terreur » dans la population constituaient une technique de contrôle social.

\section{Concevoir le désordre mondial, premiers retours sur la subversion}

La réflexion sur la subversion intérieure réapparaît au début des années 1970, parallèlement à l'émergence du « problème de l'immigration » et ce pour plusieurs raisons. En premier lieu, il s'agit pour la pensée politico-militaire de comprendre les transformations majeures que subit la géopolitique, notamment l'essor diplomatique du tiers-monde et les vastes crises énergétiques, économiques et stratégiques consécutives à la guerre de Kippour. Pour interpréter ces bouleversements, l'ordre du discours politico-militaire autorise certains courants de pensée relégués à promouvoir des modèles marginalisés. Dès la présidence de Georges Pompidou et le lessivage du SDECE ${ }^{11}$, une coopéra-

11. SDECE : Service de documentation extérieure et de contre-espionnage. 
tion poussée, notamment en termes de partage de connaissances avec les Britanniques pour l'expérimentation de la contre-subversion face aux républicains irlandais, avait autorisé les tenants de la subversion à réapparaître dans l'ombre de Frank Kitson, l'un des théoriciens principaux de la version anglosaxonne de la contre-subversion. L'arrivée au pouvoir de Valéry Giscard d'Estaing et de Michel Poniatowski inaugure le dépassement de cette pensée d'Etat incarnée par Jacques Foccart et Charles De Gaulle qui avait restreint l'emploi de l'arme contre-subversive à certains domaines (l'influence extérieure et la protection du président et de son parti notamment). Autour du nouveau président et de son ministre de l'Intérieur, passés tous deux par l'ENORSEM 12 lorsqu'elle était dirigée par le colonel Lacheroy, les réseaux de la subversion voient leur ségrégation restreinte. Au gré des amnisties successives en direction des putschistes de la fin de la guerre d'Algérie ${ }^{13}$, les réseaux dominant la production de la pensée militaire vont se réorganiser et redistribuer une légitimité relative aux promoteurs des théories contre-subversives ${ }^{14}$. La grande majorité d'entre eux a vécu les événements de 1968 comme une menace de destruction de la France par la «chienlit». Le parcours de Valéry Giscard d'Estaing représente assez bien l'accession aux sphères de commandement d'une génération marginalisée sous Charles De Gaulle et décidée à redresser une France assaillie par une série de subversions intérieures, comme le gauchisme, la drogue ou la délinquance. Les théories contre-subversives font leur premier retour en force dans la pensée militaire officielle du contrôle intérieur à cette occasion. Le général de Favitski, pour son discours de clôture de la 86 ${ }^{\mathrm{e}}$ promotion de l'ESG, le 26 septembre $1974{ }^{15}$ explique :

"L'étude du problème de la subversion et de la lutte anti-subversive, même si elle n'a pu recevoir la place autonome qu'elle mérite, a été réintroduite dans le programme avec l'accord du CEMAT ${ }^{16}$ et a saupoudré tel ou tel des exercices existant ${ }^{17}$ ».

Il faut comprendre que le retour de la subversion s'articule aussi à l'intégration de réseaux de pensée de gauche dans les courants dominant la pensée politico-militaire. C'est ce que reconnaît Pierre Dabezies, ancien adjoint de Roger Trinquier en Algérie et entré en politique comme gaulliste de gauche. Il

12. ENORSEM : Ecole nationale des officiers de réserve de l'état-major. Voir Périès, G., Servenay D., op. cit., p. 42.

13. Les décrets du 22 mars 1963, les lois du 23 décembre 1964, du 17 juin 1966, du 31 juillet 1968 et du 16 juillet 1974 notamment.

14. Rémy Martinot-Leroy montre comment les stagiaires de l'Ecole de guerre continuent de promouvoir de manière plus ou moins officielle les théories contre-subversives pendant les années 1960. Voir Martinot-Leroy R., La Contestation de la dissuasion dans l'armée de terre : l'atome et la guerre subversive dans les travaux des officiers de l'Ecole de guerre (1962-1975), doctorat en science politique, Klein J., (dir.), 1998-1999.

15. Bulletin de l'association des amis de l'ESG, $\mathrm{n}^{\circ} 64,4^{\mathrm{e}}$ trimestre 1974, p. 13.

16. CEMAT: Chef d'état-major de l'armée de terre.

17 . Voir Martinot-Leroy R., op. cit., p. 16. 
l'explique dans une conférence de 1976 sur «l'esprit de défense », prononcée à l'Ecole militaire. Cette gauche militariste n'est effectivement pas entravée par l'héritage gaulliste, elle reconduit la figure de la menace globale soviétique et une partie adhère largement au paradigme contre-subversif. Au cœur de ces penseurs de gauche du contrôle du territoire et de la lutte contre-subversive, on trouve notamment Charles Hernu, Jean-Pierre Chevènement et François Mitterrand 18. Le migrant postcolonial apparait ainsi dans ce contexte de bouillonnement de la pensée politico-militaire comme une figure de concorde et de synthèse entre discours de la dissuasion et discours de la subversion. Il permet de trouver un point de consensus entre une nouvelle pensée politico-militaire de gauche et les courants héritiers d'une position hégémonique sur le champ.

L'arrivée de la gauche au gouvernement en 1981 et la réintégration par Mitterrand des derniers putschistes et partisans OAS de l'Algérie vont accentuer ce phénomène. Ainsi, après les réhabilitations précédentes, après avoir déposé une proposition de loi prévoyant «la réintégration de plein droit dans les fonctions, emplois publics, offices publics ou ministériels ainsi que les divers droits à pension des anciens de l'OAS en 1966 ", en novembre 1982, François Mitterrand fait réintégrer dans leurs droits les huit derniers généraux putschistes, comme cela avait été prévu pendant la campagne en direction des milieux pieds-noirs et de l'armée représentée, dans ce courant, par le général Jeannou Lacaze ${ }^{19}$, un ancien du service action du SDECE et des structures de « contre-terrorisme » issues de la guerre d'Algérie.

L'ouvrage du général Jean Delaunay, un ancien d'Indochine et d'Algérie, paru en 1985 et intitulé La Foudre et le Cancer 20, constitue ainsi l'acte fondateur du retour officiel des théories contre-subversives dans le champ de la pensée politico-militaire légitime. Celui-ci y promeut, tout en conservant la doctrine de la dissuasion face au péril nucléaire ( la foudre »), la restauration des techniques psychologiques visant à amener la population à se protéger des nouvelles subversions («le cancer») que représenterait le péril identitaire et les risques de submersion véhiculés par l'immigration postcoloniale, que l'on qualifie assez généralement, depuis la fin des années 1970, d'«invasion» ou de «colonisation inversée » 21 . Le sous-titre est assez explicite : "Pour le réveil des valeurs fondamenta-

18. Le premier, spécialiste des questions de défense au PS depuis le début des années 1970 et ministre de la Défense sous les gouvernements Pierre Maurois et Laurent Fabius a fait paraître en 1975 un ouvrage livrant ses réflexions sur la nécessité de faire fusionner le soldat et le citoyen. Le second, dans un ouvrage co-écrit avec Pierre Messmer, paru en 1977, Le Service militaire, reprenait les thèses du général Beaufre sur le « syncrétisme doctrinal », c'est-à-dire l'idée d'allier doctrine de la dissuasion et réflexions sur la lutte contre subversive.

19. Voir Guisnel J., Les Généraux. Enquête sur le pouvoir militaire en France, Paris, La Découverte, 1990, fin du chap. 3 « les généraux et la politique »,pp. 66-78.

20. Voir Delaunay J., La Foudre et le Cancer. Pour le réveil des valeurs fondamentales face à l'atome et à la subversion. La guerre se gagne en temps de paix, Paris, Pygmalion, 1985, rééd. 1997, avec postface de l'auteur.

21. L'ancien Premier ministre Michel Debré rédige en 1983 un texte qui figurera dans les dossiers 
les face à l'atome et à la subversion. La guerre se gagne en temps de paix ». Malgré sa démission de l'armée en 1983, le général reçoit, pour cet ouvrage, le prix Vauban 1986 de l'IHEDN, ce qui indique encore la présence de plus en plus importante des tenants de la subversion dans les hautes études de défense.

\section{Régénérer la subversion après la disparition de la menace soviétique}

La disparition de l'URSS, en privant l'institution médiatico-sécuritaire et les institutions politiques de l'ennemi global sur lequel elles s'étaient appuyées, va autoriser de nouveaux réseaux, au début des années 1990, à travers la légitimation de la catégorie de «nouvelles menaces », à promouvoir une conception globale rénovée. S'ouvre ainsi une sorte de far-west sécuritaire à conquérir pour les réseaux coopérant à la restauration de la subversion. Durant la décennie 1990, les réseaux médiatico-sécuritaires d'où proviennent ces spécialistes de l'insécurité, des violences urbaines, du terrorisme international, du péril identitaire, etc. se stabilisent et renforcent leurs solidarités, évoluant pour certains depuis des positions périphériques vers des positions centrales et surélevées, dans de multiples secteurs du champ. Depuis ce multi-positionnement s'opère la construction d'une figure de l'ennemi intérieur global et transversal susceptible de légitimer la mise en œuvre de nouveaux dispositifs sécuritaires. Pour cause, ces dispositifs remobilisent un grand nombre d'éléments de la technologie contre-subversive. La notion de zone grise y est décisive. Elle permet d'intégrer ces territoires intérieurs considérés comme milieux de production de la menace postcoloniale que sont les quartiers populaires, à la théorie des territoires transnationaux de non-droit. Suivant le même processus que la doctrine de la guerre révolutionnaire, le modèle sécuritaire tend à l'importation pour le contrôle intérieur et sur l'ensemble de la population, des outils de la guerre coloniale. Il comporte une dynamique d'indistinction et de fusion des domaines militaires et policiers. Cette métamorphose de la structure du contrôle social s'observe à travers l'alliance de plus en plus poussée entre des réseaux médiatico-sécuritaires (chargés de la diffusion des imaginaires sur l'ennemi et les moyens de le soumettre), des réseaux politico-militaires (chargés de les constituer, de les autoriser et de les traduire en marchés d'Etat) et, finalement, des réseaux industriels et financiers désireux de conquérir le marché sécuritaire découvert par une privatisation encore relative du contrôle intérieur. Comme le montre un article du lieutenant-colonel Grégoire de Saint-Quentin dans la principale revue de la communauté politico-militaire en 1997, il est désormais largement légitime de convoquer le répertoire de la doctrine de la guerre révolutionnaire pour envisager le contrôle sécuritaire local («les cités») ou global (les « zones grises») 22.

d'information de l'IHEDN dès la session suivante. Il est intitulé L'immigration est-elle une invasion ?, question à laquelle l'auteur répond par l'affirmative, en y conjuguant une réflexion sur l'essor de la délinquance des immigrés qu'il désigne comme une « situation explosive ».

22. Le lieutenant-colonel de Saint-Quentin a notamment été chargé de l'instruction de pelotons CRAP au Rwanda avant le génocide. Voir de Saint-Quentin G., « Retour à la guerre révolutionnaire ? ", Défense nationale, octobre 1997, p. 105. 


\section{La menace migratoire. Une perspective de défense globale}

Pour comprendre le rôle de la menace migratoire dans la relégitimation $\mathrm{du}$ répertoire contre-subversif, il faut revenir sur la doctrine de la défense globale. Cette structure discursive détient une fonction centrale dans le processus d'ethnicisation des menaces.

\section{Penser les menaces dans le cadre de la défense globale}

La construction de l'immigration comme menace dans la pensée militaire est liée parallèlement à l'institution préalable de la doctrine de la défense globale. L'ordonnance du 7 janvier 1959 qui la régit à partir de la Constitution de la Ve République est forgée dans le même contexte sociohistorique, imaginaire et discursif que la doctrine de la guerre révolutionnaire. Croisant les problématiques de la Guerre froide, celles de la guerre totale et celles du terrain colonial, faisant fusionner les corps de l'ennemi fellagha, civil, intérieur, infériorisé, racialisé, et celui de l'ennemi communiste, civil ou militaire, intérieur et extérieur, global, encerclant, il s'agit d'y justifier comme nécessité de ne plus distinguer l'intérieur de l'extérieur, le civil du militaire, le temps de paix du temps de guerre. La logique intrinsèque de cette pensée institue une forme de mise en état de guerre permanent, autorisant l'intervention du militaire dans la société, une fusion du contrôle policier de la population et des techniques militaires de l'encadrement et du commandement en territoire et en période d'exception. Au début des années 1970, au moment où apparaît la menace migratoire dans la pensée politico-militaire, la défense globale est encore largement légitimée par les métaphores corporelles et chirurgicales qui associent la menace à une agression virale du corps national. Un article de P. Lefebvre, universitaire en fonction au SGDN, paru dans Défense nationale en 1978 fait la synthèse de ce qu'il est alors convenu d'appeler la «défense globale» ${ }^{23}$. La rédaction de Défense nationale explique qu'elle emprunte «cette assimilation de la défense à un organisme vivant à l'allocution $d u$ président Georges Pompidou, le 3 novembre 1969 à l'IHEDN» : "Toute défense nationale dépend du ressort moral, de la résolution, de la volonté de vivre qui animent un peuple. Comme un malade dont on dit qu'il a mauvais moral, une nation qui s'abandonne est condamnée ». Elle explicite cette persistance d'une sémiologie contre-subversive dans le concept de défense globale comme "propre à éclairer la notion de menace globale qui recouvre l'ensemble des agressions de tous ordres, économiques, culturelles, mais aussi morales qui, si nous n'y prenons garde, peuvent ruiner la nation ». Ce qui diffère principalement de la «menace globale» envisagée auparavant dans le courant des guerres coloniales, c'est que le vecteur principal, le monde communiste, tend désormais à être remplacé par une nébuleuse de menaces plus ou moins formelles, au sein desquelles figure le monde communiste, à côté du terrorisme, de l'individualisme, du matérialisme, de l'islam,

23. Voir Lefebvre P., « La menace globale », Défense nationale, février 1978, p. 45. 
de la démographie... Cette diversification ne bouleverse pas les conceptions dominantes de la défense globale, bien au contraire, elle permet de reconduire les principes de l'indistinction du civil et du militaire, de la paix et de la guerre et la stigmatisation d'une menace intérieure mue depuis l'extérieur, à travers un nouveau discours sur la population en général : la sécurité. La construction de l'immigration comme menace va constituer, de ce point de vue, l'un des axes de cette reconduction de la défense globale face au déclin de la menace soviétique. Elle va répondre à un désir de globalité dans la figuration de l'ennemi, qui simplifie et synthétise les menaces particulières autour d'un concept clair et pragmatique : l'ennemi est partout, caché dans la population, à l'intérieur comme à l'extérieur $d u$ territoire, il se confond avec elle; pour le réduire, il faut amener la population à s'auto-contrôler, à opérer elle-même son immunisation, en lui faisant prendre conscience $d u$ danger qu'elle court. C'est sur ce principe - considérer la population comme le milieu de prolifération de la subversion et, en même temps, comme le corps à protéger - que la guerre coloniale-révolutionnaire a constitué l'un des laboratoires principaux de conception de la pensée sécuritaire.

\section{La construction $d u$ "problème de l'immigration "}

Apparaissant au début des années 1970 comme une menace démographique avec l'émergence du tiers-monde en tant qu'acteur transnational, puis comme une menace économique à mesure que la crise économique est conçue comme telle, le migrant originaire du monde arabe devient l'idéal-type d'une menace géopolitique, religieuse et identitaire à partir de l'intensification des antagonismes internationaux au Proche-Orient puis de la révolution khomeyniste en Iran.

C'est sous l'autorité de Pierre Chaban-Delmas et de Michel Poniatowski que sont mises en place les premières lois visant à suspendre l'immigration et qu'est traduite en termes politiques, puis en dispositifs de contrôle, la notion de sécurité. Un texte de Paul Dijoud, le secrétaire d'Etat aux travailleurs immigrés, constitue l'énoncé fondateur $\mathrm{du}$ " problème de l'immigration » dans la pensée de la défense globale. Il est publié en 1976 dans Défense nationale sous le titre «La France et les immigrés » 24 . Premier article de la revue, il synthétise un très grand nombre des dispositifs visant à stigmatiser une menace globale issue du corps immigré, décrié jusque-là comme le support d'enjeux qui le dépassaient et désormais comme une menace en soi. Il frappe ce modèle du sceau de l'autorité officielle de la pensée d'Etat. Ce texte était censé légitimer auprès du monde politico-militaire les lois de 1973-1974 visant à restreindre, puis à stopper l'entrée de travailleurs immigrés en provenance des anciennes colonies.

Au cours des années 1980 et suite à l'invention des « secondes générations » qui permettent de situer, parmi les Français, une sorte de cinquième colonne

24 . Dijoud P., « La France et les immigrés », Défense nationale, mai 1976, p. 11. 
orientale au cœur de l'Occident, le migrant se pare des stigmates de l'ennemi pauvre, puis de ceux visant à qualifier une menace politique et sociale (« le jeune désœuvré », « le délinquant », « le casseur », etc.). Dans le même temps, la figure $\mathrm{du}$ «terroriste rouge » qui avait recouvert celle du «terroriste musulman » depuis la fin de la guerre d'Algérie, est ré-ethnicisée, pour désigner un potentiel de conversion au terrorisme international islamiste dans la population arabomusulmane en France, catégorie permettant elle-même de réinstituer une fracture socio-raciale dans la conception de la nation, dispositif essentiel qui caractérisait la structure coloniale et les schèmes de pensée contre-subversifs.

\section{L'ethnicisation des menaces}

La menace terroriste prend, dans le courant de la décennie 1980, des dimensions très importantes au sein de la pensée politico-militaire, et l'enjeu de la lutte entre les différents courants de pensée vise moins à savoir s'il s'agit d'une menace globale - ce qui est largement partagé -, qu'à connaître son rapport en qualité et en quantité à la menace soviétique. Ce sont de nouveaux réseaux, les spécialistes de la sécurité internationale qui imposent leurs conceptions lorsque la menace soviétique disparaît. Ils s'imposent ainsi eux-mêmes dans le champ de production du contrôle. Les attentats de 1986 sur le territoire français semblent avoir joué un rôle décisif dans le cadre de l'ethnicisation de la figure du « terroriste international ", avant même la chute de l'URSS. Ils leurs servent de marchepied. En les attribuant à une obscure nébuleuse islamiste, ces nouveaux réseaux de "spécialistes de la sécurité internationale », qui sont, pour certains de leurs acteurs, des anciens de la lutte contre-subversive en Algérie, et qui en connaissent les axes théoriques pour l'immense majorité, vont pouvoir se légitimer progressivement puis conquérir des positions d'autorité dans le champ. Cette problématique commence alors à s'articuler aux réflexions sur le contrôle du territoire, des frontières notamment, ainsi que sur l'encadrement des étrangers et de leur circulation. La décennie 1980 voit s'instituer la conjugaison des figures du «terroriste » et de l'étranger, par le partage d'un stigmate de suspicion vis-à-vis de l'allégeance nationale. La question de l'allégeance des binationaux, des clandestins, des étrangers en général, d'un point de vue culturel, religieux, politique ou dans le cadre du maintient de l'ordre va déterminer l'évolution du débat sur la réforme du Code de la nationalité. Il s'agit, pour ce débat, de redéfinir le statut du national et du sol, ainsi que des modalités d'acquisition de la nationalité française en fonction de la reconnaissance de ces menaces transversales que sont le «terrorisme », le « péril identitaire », la «délinquance » ou l'« islam »; ou comment s'assurer de ne pas naturaliser des subversifs. A travers la question de la binationalité on reconstruit, à mi-chemin entre « le clandestin » et le «Français d'origine immigrée », une figure du «faux-Français », du Français suspect de défaut d'allégeance.

La promotion de la «menace islamiste » désigne le groupe des « musulmans en France » comme un milieu susceptible d'abriter les germes d'une sub- 
version globale. Son articulation à la menace terroriste amène ces réseaux d'auteurs à réfléchir sur les états d'exception et les manières de mettre en œuvre une défense globale requérant, en période de paix, des méthodes de guerre contre des populations civiles, nationales ou non. Elle va ainsi pouvoir piocher dans le répertoire technique colonial dont la particularité avait été de réfléchir à l'encadrement d'une menace globale incrustée dans la population musulmane, d'une technique d'exception pour une population d'exception. On assiste ainsi à la constitution d'une pensée politique et militaire, d'Etat et privée, centrée sur la reconnaissance d'une menace transnationale prenant appui sur une cinquième colonne socio-ethnicisée, cachée dans la population musulmane, où que celle-ci se trouve dans le monde.

\section{L’ennemi transversal. Construire la sécurité internationale}

La construction de l'immigration comme menace s'inscrit parallèlement dans un processus de construction internationale d'un ennemi commun et transversal. Celui-ci s'opère à travers l'institution de l'antiterrorisme comme stratégie globale et du migrant comme suspect par nature.

\section{L'immigration comme menace transversale}

Avec la disparition de l'ennemi soviétique, le corps immigré va pouvoir apparaitre comme le support de reconstruction d'un ennemi intérieur, socio-ethnique, furtif, global. A la différence de l'indigène-partisan des guerres d'Indochine et d'Algérie, cet ennemi intérieur est désormais conçu comme transversal, et sa désignation permet de justifier la coopération de réseaux médiatiques, économiques, politiques et sécuritaires autour de la production de contrôle. La première guerre d'Irak, puis les attentats de 1995 sur le territoire français vont ainsi constituer le socle sur lequel instituer une prospective de la guerre civile postcoloniale, permettant aux entrepreneurs du contrôle de transformer le marché de la menace intérieure en économie de guerre permanente. Des théories de la guerre civile mondiale apparues au cours des années 1950 vont ainsi être remobilisées de manière à articuler à la notion de zone grise, celles de chaos mondial et de nouvelles menaces transversales. Si l'étude des courants dominant la pensée militaire française permet d'observer, avec le 11 septembre 2001, une intensification de la coopération internationale autour du contrôle des frontières, il ne semble pas qu'elle se transforme réellement. La base du modèle sécuritaire de réduction de l'ennemi intérieur global est en effet mise en ouvre depuis les premières réactivations du plan Vigipirate en 1993-1994. Le 11 septembre ne semble, lui, fonctionner que comme le stimulateur d'une dynamique déjà expérimentée depuis plus d'une décennie selon une gradation du temps de la crise permanente, les innovations en termes de contrôle et de restriction des libertés publiques s'empilant par effet de cliquet. Il semble que la figure de l'ennemi socio-ethnique et transversal se stabilise autour de 1995, après la médiatisation des attentats attribués au FIS. La figure de Khaled Kelkal synthétise, de ce point de vue très précisément, la représentation 
dominante de l'ennemi intérieur dans la pensée politico-militaire française de l'après-Guerre froide : un jeune mâle musulman et d'origine étrangère, issu des quartiers populaires dont la foi et/ou la délinquance constitueraient le terreau du terrorisme islamiste et des violences urbaines. La répression des révoltes de l'automne 2005 a déterminé de la même manière l'intensification et la diversification de mécanismes amorcés et expérimentés depuis déjà une décennie. Les quartiers populaires ségrégués servaient de territoire d'expérimentation pour l'importation de la guerre urbaine et du contrôle des foules dans le maintien de l'ordre, depuis les émentes de Villeurbanne au début des années 1990. Leur traitement médiaticopolitique aura permis de légitimer l'émulation d'un processus de fusion des techniques policières et militaires dans le quadrillage des territoires d'exception. Cette dynamique s'inscrivait déjà dans la redéfinition et le redéploiement de la gendarmerie - structure de statut militaire - et une superposition des maillages de sécurité et de défense sur les zones grises. Ces mouvements d'exportation et d'importation, d'expérimentation collective des nouveaux contextes de la guerre (la bataille d'Alger, l'Irlande, l'Irak, la Palestine, etc.), traduisent un phénomène : le modèle sécuritaire revendique l'élaboration d'une souveraineté transnationale notamment et en tant qu'il est le produit d'une fabrique transnationale, qu'il permet d'associer des réseaux économiques, politiques, médiatiques industriels et militaires autour de l'ouverture d'un marché planétaire à conquérir.

\section{Vigipirate, sécuriser le territoire}

Dès 1974, les rapports de comité IHEDN et la revue Défense invitent à considérer l'évolution de la notion de défense vers celle de sécurité, pour insister sur la permanence et la globalité du contrôle à mettre en place ${ }^{25}$. La notion de sécurité, posant la population et le territoire à la fois comme des objets à protéger et comme les milieux de production d'une menace intérieure, vise d'une certaine manière à généraliser dans le temps, l'espace et les catégories, des techniques de contrôle conçues comme exceptionnelles ${ }^{26}$. C'est ainsi, sous le président Giscard d'Estaing, selon la même dynamique visant à fermer les frontières et inciter les migrants nord-africains à quitter le territoire, qu'est instituée l'idée selon laquelle "la sécurité est la première des libertés », que sont votées les lois anti-gang et qu'est créé le plan Vigipirate en 1978. Cette première idée va diriger l'ensemble du paradigme visant à légitimer le modèle sécuritaire dans les décennies suivantes. Les lois anti-gang constituent, elles, le tout premier retour en France et pour l'intérieur d'un répertoire désormais « occidental » de la contre-subversion. Elles renvoient très précisément aux dispositifs anti-gang conçus par Frank Kitson pour l'Irlande du Nord à partir

25. Voir Schlumberger J., «Rapport de comité : Défense nationale et sécurité en Europe », Défense, $\mathrm{n}^{\circ} 1$, octobre 1974, p. 13.

26. Voir par exemple les travaux de Barry Buzan et Ole Waever posant l'exceptionnalisation comme le symptôme de la sécurisation : Buzan B., Waever O., de Wilde J., Security, a neww framework for analysis, Londres, Line Rienner, 1998. 
d'une rénovation des dispositifs algériens de Roger Trinquier. La translation s'opère en prenant appui sur la désignation d'un ennemi intérieur furtif, traversant l'ensemble du corps social, le délinquant, le casseur, le gauchiste... Il s'agirait selon les concepteurs de l'anti-gang à la française d'appliquer au maintien de l'ordre les méthodes de l'anti-terrorisme, à l'époque encore considérées comme appartenant au domaine de la guerre. Ainsi s'amorce une conception du quadrillage urbain métropolitain à partir de l'apport technologique de l'antiterrorisme en guerre coloniale. Le plan Vigipirate est conçu en 1978 pour faire face à une menace globale véhiculée par une « internationale terroriste » incarnée par des «faux réfugiés politiques » et des «clandestins ». Il signale l'institution, dès la fin des années 1970, de l'amalgame du migrant et du «terroriste ». De surcroît, comme tout ce qui a trait au fait militaire, nucléaire ou africain dans la conception de la défense globale et selon la constitution de la Ve République, le plan Vigipirate relève du domaine présidentiel. Avisé par des "services spécialisés ", qu'il juge légitimes dans leur appréciation de la menace, le président décide de l'activation du plan, comme d'un état d'exception supplémentaire, complétant le répertoire des leviers à disposition du souverain pour suspendre le contrôle juridique et législatif. Classé confidentiel défense, celui-ci n'est pas publié et ne doit sa légitimité juridique qu'à l'ordonnance du 7 janvier 1959, qui organise la défense nationale sur la base de la défense globale, c'est-à-dire au bon vouloir du chef des armées. Le plan a été actualisé à trois reprises, en juillet 1995, juin 2002 et mars 2003. Il est régi par le nouveau code de la défense depuis sa publication, en avril 2007. Son évolution est indissociable de l'instauration du terrorisme comme menace globale, puis l'intensification et la généralisation de la lutte contre l'immigration, d'abord par le contrôle des frontières, puis par le quadrillage du territoire. Il a été déployé pour la première fois en 1991, dans le cadre de la guerre du Golfe et est devenu, depuis 1996, une sorte de disposition d'urgence permanente, fondée sur l'idée que la vie de l'Etat et de la population est désormais constamment en jeu. Le plan Vigipirate n’a pas été levé depuis, et est régulièrement réactivé en position « renforcée », dont l'effet principal est l'intensification de la militarisation du quadrillage urbain et de l'emploi de l'armée dans une fonction policière. L'indistinction relative entre temps de paix et temps de guerre avait nourri la notion de crise ; il semble que l'on passe, à partir de l'activation permanente de Vigipirate, à une temporalité de la crise permanente mais nivelée et graduée ${ }^{27}$. Le code couleur utilisé pour signifier le degré de la menace et la puissance de contrôle à mettre en jeu, (jaune, orange, rouge, écarlate) réactive la métaphore nosologique de l'infection virale et du traitement chirurgical. La plupart des auteurs politico-militaires le conçoivent comme un instrument de dissuasion face au terrorisme et surtout de réduction du sentiment d'insécurité, cependant, une partie très

27. Voir de Andrade A, «La distinction temps de paix / temps de guerre en droit pénal militaire : quelques éléments de compréhension ", Champs de Mars, Cahiers du C2SD, Paris, La Documentation française, 2002, p. 161. 
minoritaire mais non négligeable admet qu'il a essentiellement pour fonction une sorte de pacification permanente du territoire ${ }^{28}$.

Le plan prévoit également le partage et la diffusion d'une culture de sécurité. Il repose en effet sur un principe de responsabilité partagée de la sécurité : chacun devant prendre en compte les risques, du simple citoyen aux services chargés d'intervenir contre le terrorisme. Conçu puis promu par de nombreux tenants de la contre-subversion, il hérite d'un nombre important d'autres dispositifs issus des pensées contre-subversives. Le plan définit ainsi l'emploi du militaire dans le contrôle intérieur pour encadrer la population comme milieu de propagation d'une menace terroriste. Il incite à promouvoir une action psychologique sur la population pour l'amener à isoler les subversifs et à s'auto-surveiller. Il institue des structures de renseignement permanentes dans la population. L'élaboration du plan Vigipirate a constitué le lieu principal de transmutation de la pensée contre-subversive en pensée sécuritaire.

\section{Construire la sécurité internationale face à un adversaire en commun}

Le migrant semble constituer une figure transversale permettant d'activer une coopération internationale pour le contrôle des frontières, un projet de renforcement de la souveraineté des Etats d'accueil sur le territoire, et donc un levier politico-économique sur les Etats de provenance qui appartiennent, pour la plupart, au monde colonisé. Articulé à la figure de l'étranger et du clandestin au début des années 1970, le migrant permet ainsi de convoquer des discours sur la sécurité intérieure, en termes identitaires, juridiques ou économiques.

Nous avons analysé le cas français, mais des travaux comme ceux d'Ayse Ceyhan ou de Philippe Bonditti ${ }^{29}$ permettent d'observer des phénomènes identiques aux Etats-Unis. L'articulation des figures du migrant pauvre et de celles du « terroriste clandestin » va déterminer la mise en ouvre des premières structures antiterroristes transnationales à la fin des années 1970 et des premiers accords entre pays d'accueil pour une coopération dans la production de contrôle sur un territoire commun. On peut, à la lecture de travaux sur la genèse de l'antiterrorisme international, concevoir l'idée que ces plans de défense ont constitué pour l'ensemble des puissances engagées dans la lutte contre le terrorisme, un même type de laboratoire commun. En France comme aux Etats-Unis, toute l'histoire de l'anti-terrorisme est liée à celle du contrôle des frontières et à la surveillance de la population comme milieu de prolifération d'une subversion exogène.

28. Voir par exemple l'article du gendarme Christian Choquet dans le $\mathrm{n}^{\circ} 44$ de Cultures $\mathcal{E}$ Conflits, op. cit.

29. Voir notamment leurs articles respectifs dans le $n^{\circ} 44$ de Cultures E Conflits consacré à « Défense et identités, un contexte sécuritaire global ? ", op. cit. 
En France, le «problème de l'immigration » apparaît dans les travaux de l'IHEDN au contact de documents visant à expliquer la nécessité de fermer les frontières, d'en faire la base d'une coopération européenne, c'est-à-dire le support de construction de la Communauté. On y observe une attention constante des courants français pour le « problème noir » américain comme prospective de la guerre civile en France ${ }^{30}$. La problématique est liée à celle de la « sur-délinquance nord-africaine » à un moment où l'incivilité et la délinquance sont posées comme de nouveaux enjeux de société, de nouveaux domaines intéressant la défense globale. Entre 1986 et 1989, c'est la même dynamique qui organise ainsi, dans les discours de Charles Pasqua devant l'IHEDN, la représentation d'une menace terroriste et celle d'une menace migratoire. Cette articulation lui permet de justifier un nouveau plan de défense intérieure du territoire ainsi que l'urgence de réformer le code de la nationalité : l'immigration postcoloniale incarnerait un milieu de prolifération pour l'ennemi intérieur, et les cinquièmes colonnes d'une nouvelle menace globale, omnisciente, omniprésente, omnipotente ${ }^{31}$.

La construction de la menace migratoire est coextensive à l'élaboration d'un discours sur l'ordre international et la coopération dans la production de contrôle. Avec les années 1990, on voit s'imposer dans les travaux des auditeurs de l'IHEDN comme dans la presse de défense, autour de la problématique du contrôle intérieur et des frontières, des séries d'analyses visant à faire des retours d'expérience (RETEX) collectifs sur la pacification de Bagdad, de Srebrenica, d'Abdidjan ou de Clichy-sous-bois. Tous concluent sur la nécessité de lier la lutte contre les ennemis intérieurs sur le plan national au contrôle international de l'immigration. Le contrôle global des frontières et la fusion du militaire et du policier dans le domaine du maintien de l'ordre trouvent un terrain commun en la figure de l'ennemi transversal socio-ethnique. A la fin des émentes de 2005, qu'il avait attribuées à l'immigration, à l'islamisme et même à la polygamie, le ministre de l'Intérieur Nicolas Sarkozy invitait, sur ce principe, en décembre 2005 à Paris, les chefs de la police israélienne Gideon Ezra et Moshe Karadi pour partager les savoir-faire français et israéliens en matière de contrôle des foules et de maintien de l'ordre en milieu urbain ${ }^{32}$.

\section{Les héritages contre-subversifs du modèle sécuritaire}

Nous avons synthétisé les modèles contre-subversifs et les modèles sécuritaires à travers cinq propositions. Ceci permet de mettre en évidence les axes

30. Voir Rossi M., «Le problème noir aux Etats-Unis et son impact sur la politique américaine », Défense nationale, janvier 1978, p. 81.

31. Voir l'article de Seth Cropsey dans Défense nationale présentant la conception internationale d'un appareil sécuritaire « omniscient, omniprésent et omnipotent » comme une question de survie face aux nouvelles formes de la menace : Cropsey S., « Omniscient, omniprésent, omnipotent: $\mathrm{O}^{3}$ », Défense nationale, avril 1997. Seth Cropsey a notamment été le principal assistant du secrétaire de la Défense pour les opérations spéciales et les conflits de basse intensité sous la présidence de Georges Bush père.

32. Voir Haaretz, 12 décembre 2005. 
discursifs issus de la guerre coloniale qui ont servi de répertoire technique pour la pensée sécuritaire. On y observe des reconductions strictes, des reformulations plus ou moins profondes, ainsi que des réagencements.

\begin{tabular}{|l|l|}
\hline $\begin{array}{l}\text { Doctrine de la guerre } \\
\text { révolutionnaire }\end{array}$ & Modèle sécuritaire \\
\hline $\begin{array}{l}\text { 1. Les populations colonisées sont } \\
\text { des milieux de prolifération de la } \\
\text { menace communiste à contrôler }\end{array}$ & $\begin{array}{l}\text { 1. La population est le milieu de } \\
\text { prolifération de la menace et l'enjeu } \\
\text { même du contrôle. }\end{array}$ \\
\hline $\begin{array}{l}\text { 2. Le renseignement doit permettre } \\
\text { de faire apparaitre les hiérarchies } \\
\text { parallèles adverses à tenir, détruire } \\
\text { ou remplacer. }\end{array}$ & $\begin{array}{l}\text { 2. Le renseignement, face aux nou- } \\
\text { velles menaces, doit permettre de } \\
\text { tout voir, tout savoir, tout prévoir. }\end{array}$ \\
\hline $\begin{array}{l}\text { 3. La terreur permet de tenir la pop-3. Faire prendre conscience à la } \\
\text { population, de la menace globale. }\end{array}$ \\
ulation. & $\begin{array}{l}\text { 4. Former les institutions (médias, } \\
\text { écoles...) à la promotion de l'esprit } \\
\text { de défense, pour immuniser la pop- } \\
\text { ulation. }\end{array}$ \\
\hline $\begin{array}{l}\text { 4. Action psychologique pour } \\
\text { retourner la population tenue. }\end{array}$ \\
\hline $\begin{array}{l}\text { 5. Quadrillage militaro-policier } \\
\text { (DPU /DIT) de l'espace urbain. }\end{array}$ & $\begin{array}{l}\text { 5. Quadrillage nivelé mais perma- } \\
\text { nent, fusion des dispositifs militaires } \\
\text { et policiers en ville. }\end{array}$ \\
\hline
\end{tabular}

La lutte transnationale contre le péril soviétique a fourni les bases d'une expérimentation conjointe de la lutte contre le terrorisme et l'immigration. Cette dernière a fourni l'axe principal de la production internationale du contrôle dans l'après-Guerre froide. La construction de l'immigration comme menace a servi de support au retour de la pensée contre-subversive dans le répertoire du contrôle intérieur et à la cohésion de la pensée de la défense globale face à la reconnaissance du désordre mondial. Elle a également servi de support à la justification de l'indistinction relative du militaire et du policier et la privatisation du contrôle constitutives de la mise en place du modèle sécuritaire. L'enchevêtrement des discours identitaires et des discours sécuritaires s'inscrit dans la reconduction d'une pensée métaphorique centrale pour les théories contre-subversives : la nation est un corps qu'il faudrait immuniser contre une subversion de type virale, par la diffusion de l'esprit de défense et 
l'encadrement de ce corps par l'institution militaire, se présentant comme le chirurgien des affections mortelles pour cette communauté imaginée qu'elle appelle nation.

Une généalogie de l'ennemi intérieur dans la pensée politico-militaire française montre comment la guerre coloniale a constitué un lieu de conception et d'élaboration de la pensée et du contrôle sécuritaire. Les régénérations de la contre-guérilla dans l'antiterrorisme peuvent expliquer la généralisation dans le temps et dans l'espace postcoloniaux, de pratiques de contrôle d'exception et de techniques de guerre. 